\title{
Pre-Morbid Risk Factors for Amyotrophic Lateral Sclerosis: Prospective Cohort Study
}

\author{
G David Batty (D) \\ Catharine R Gale ${ }^{2}$ \\ 'Department of Epidemiology and Public \\ Health, University College London, \\ London, UK; ${ }^{2}$ Medical Research Council \\ Lifecourse Epidemiology Unit, University \\ of Southampton, Southampton, UK
}

\begin{abstract}
Aim: In the absence of effective treatments for amyotrophic lateral sclerosis (ALS), a neurodegenerative disorder with high case fatality, there is a clear need to identify its primary risk factors.

Methods: UK Biobank is a prospective cohort study in which baseline data were captured between 2006 and 2010 in 502,649 participants aged 37 to 73 years. Follow-up for ALS hospitalisations and death was made via national registries.

Results: Eleven years of event surveillance gave rise to 301 hospitalisations and 279 deaths due to ALS. After adjustment for selected confounding factors, being older (hazard ratio per 10 year increase; 95\% confidence interval: 1.92; 1.58, 2.33) and male $(1.37 ; 1.00,1.87)$ were associated with elevated rates of hospitalisation for ALS. Similar effects were apparent when death ascribed to the disorder was the outcome of interest. Of the remaining 23 social, biological, and behavioural risk indices, however, there was only a suggestion that taller people experienced an increased risk of hospitalisation (per SD increase: $1.31 ; 1.09,1.59)$.
\end{abstract}

Conclusion: In the present, large-scale study, other than well known associations, we did not find convincing evidence of links with ALS for other risk indices.

Keywords: risk factors, amyotrophic lateral sclerosis, cohort study, UK Biobank

\section{Introduction}

Amyotrophic lateral sclerosis (ALS), also known as motor neuron disease, involves the unabated degeneration of nerve cells responsible for voluntary muscle movement. With there being no effective treatment, in most cases, death, typically from respiratory failure, commonly occurs within 3 years of symptom emergence. ${ }^{1,2}$ This brings into sharp focus the need for primary prevention research.

While marked geographical differentials in case occurrence ${ }^{3}$ implicates both genetic and non-genetic causes of ALS, a secular increase in rates over three decades ${ }^{4}$ - seemingly too rapid to be explained by changes in the gene pool - provides some circumstantial support for a role of environmental determinants. There is strong evidence to suggest being both male and older are associated with an elevation in risk, ${ }^{5}$ however, studies examining the role of modifiable environmental characteristics report inconsistent and often counter-intuitive results. Thus, although higher prediagnostic body weight appears to confer protection against ALS, ${ }^{6,7}$ cigarette smoking has revealed discordant associations, ${ }^{8,9}$ as have physical activity, ${ }^{10}$ alcohol intake, $^{11,12}$ socioeconomic status, ${ }^{13,14}$ and physical comorbidities such as diabetes, ${ }^{15,16}$ amongst many other factors. ${ }^{17}$
Correspondence: G David Batty

Department of Epidemiology and Public Health, University College London, I-19 Torrington Place, London, WCIE 6BT, UK

Email david.batty@ucl.ac.uk 
The heterogeneity of these findings may, at least in part, be explained by different methodological shortcomings across studies. Given the rarity of ALS, the most time- and cost-efficient method for identifying risk factors is the case-control study, interpretation of findings from which is complicated by recall bias and, potentially, reverse causality, such that biomedical indices, for instance, are captured in the presence of the disorder. Accordingly, we used a well-characterised prospective cohort study of around half a million people with ongoing surveillance for mortality and morbidity from ALS in order to better understand the role, if any, of awide range of modifiable biological, psychosocial, and behavioural characteristics. In particular, our purpose was to further examine some extant associations and also to relate previously untested characteristics to the occurrence of the disorder. There are, for example, reasons to anticipate associations between ALS and earlier measurements of cognitive function, psychological distress, and lung function, not least because these characteristics may be pre-clinical features of the disorder. ${ }^{18}$ Although these indices have shown prognostic value in studies of patient groups - less favourable levels are associated with adverse outcomes including disability severity and reduced life expectancy $^{19,20}$ - they are untested as risk factors for disease occurrence.

\section{Methods}

UK Biobank, a UK-wide, closed, prospective cohort study, has been described in detail. ${ }^{5}$ In brief, all people aged 40 69 years who were registered with the UK National Health Service and living a maximum of 25 miles from one of the 22 study assessment centres were invited to participate. Of the 9.2 million people asked, 503,325 study members (5.5\% response) completed a questionnaire, underwent an interview, and took part in various physical assessments. Ethical approval was obtained from the National Health Service National Research Ethics Service with all participants providing written consent. Using fully anonymised data, the present analyses did not require additional permissions. We followed STROBE guidelines for the presentation of original epidemiological research. ${ }^{21}$

\section{Assessment of Baseline Characteristics}

Ethnicity was self-reported and categorised as White, Asian, Black, Chinese, Mixed, or other ethnic group. ${ }^{22}$ A social isolation scale was derived from enquiries concerning number of people in the household, visits with friends/family, and social activities. ${ }^{23}$ For educational qualifications, we used a two category variable (college/university degree versus lower). Total annual household income before tax was self-reported. The Townsend deprivation index was our indicator of neighbourhood socioeconomic circumstances; a continuously scored variable, higher values denote greater deprivation. ${ }^{24}$ Based on national census data, scores comprise information on employment, overcrowding, and car and home ownership. Each participant was assigned a Townsend value corresponding to the postcode of their home address.

Cigarette smoking, physical activity, and alcohol consumption were all self-reported. ${ }^{25}$ Smoking status was categorised into never, former, and current; alcohol intake was grouped into daily/almost daily, three to four times a week, once or twice a week, one to three times a month, special occasions only and never; and physical activity was captured using the International Physical Activity Questionnaire short form. ${ }^{26}$ Height and weight were recorded directly and body mass index was calculated using the usual formula (weight, $\mathrm{kg} /$ height $^{2} \mathrm{~m}^{2}$ ); overweight and obesity were denoted by values $\geq 25 \mathrm{~kg} / \mathrm{m}^{2}{ }^{27}$ Self-reported physician diagnosis was collected for ALS, vascular or heart problems, diabetes, and cancer. We defined hypertension according to existing guidelines: measured systolic/diastolic blood pressure $\geq 140 / 90$ $\mathrm{mmHg}$ (two assessments) and/or self-reported use of antihypertensive medication. ${ }^{28,29}$

Forced expiratory volume in one second, a measure of pulmonary function, was quantified using spirometry with the best of three technically satisfactory exhalations used in our analyses. Handgrip strength was measured using a hydraulic hand dynamometer with the participant maximally squeezing the handle of the dynamometer while seated for 3 seconds; an average of readings from the right and left hand was used. Non-fasting venous blood, available in a sub-sample, was drawn with assaying for high-density lipoprotein cholesterol and glycated haemoglobin A1c conducted at a single laboratory. ${ }^{30}$

Study members were asked if they had ever been under the care of a psychiatrist for any mental health problem. ${ }^{31}$ Symptoms of psychological distress - anxiety, worrying, anhedonia, and depression - were measured using the four item version of the Patient Health Questionnaire (PHQ-4) ${ }^{32,33}$ in which individual items are rated on a Likert scale from 0 ("not at all") to 3 ("nearly every day") such that total scores range from 0 to 12 (higher scores denote greater distress). A score of 3 or above was used to indicate high distress in 
the present analyses. ${ }^{31}$ Neuroticism was measured with the 12item Eysenck Personality Questionnaire-Revised Short Form; ${ }^{34,35}$ again, higher scores denote higher levels. Verbal and numerical reasoning was captured using a computerized 13-item multiple-choice test with a two-minute time limit; the score was the number of correct answers. ${ }^{36}$ This test was introduced after the beginning of the baseline assessment period so data are only available for a subset of study members $(\mathrm{N}=180,914){ }^{24,37}$ Reaction time was measured using a computerized Go/No-Go "Snap" game. ${ }^{37}$ Participants were presented with electronic images of two cards. If symbols on the cards were identical, participants were instructed to immediately push the button-box using their dominant hand. The first five pairs were used as a practice with the remaining seven pairs, containing four identical cards, forming the assessment. Reaction time score was the mean time to press the button when each of these four pairs was presented. Choice reaction time correlates strongly with single mental tests that involve complex reasoning and knowledge. ${ }^{38}$

\section{Ascertainment of Amyotrophic Lateral Sclerosis Cases}

Study participants were linked to the National Health Service's Central Registry which provided vital status data on study members and, where applicable, cause of death. Linkage was also made to hospital in-patients records via the Hospital Episode Statistics, a registry of all hospitalisations in the UK. ${ }^{39}$ Using both databases, ALS was denoted by International Classification of Disease code G122.

\section{Derivation of Analytical Sample and Statistical Analyses}

To addresses concerns regarding reverse causality - the notion that ALS might influence the exposure of interest (eg, physical activity, lung function) rather than the opposite - we excluded 56 people who self-reported ALS at baseline medical examination. Additionally, to capture study members with potentially subclinical (undiagnosed) ALS, we left-censored study members such that those who were hospitalised for or died from the condition within the first 3 years of baseline were also excluded( $(\mathrm{N}=72)$ - as described, death commonly occurs within this period after symptom emergence. ${ }^{1,2}$ This resulted in an analytical sample of 502,599 people (273,454 women). To summarise risk factor-ALS associations, we computed hazard ratios with accompanying 95\% confidence intervals using Cox proportional hazards regression. ${ }^{40}$ In these analyses, we used calendar period as the time scale and study members were censored at date of hospitalisation or death from ALS, or end of follow-up (4th October 2020 for mortality, 24th November 2020 for hospitalisation) - whichever came first. We used Stata version 15 for all analyses.

\section{Results}

Event surveillance gave rise to 301 hospitalisations (mean 11.8 years of follow-up) and 279 deaths (11.4 years) due to ALS. In Tables 1 (hospitalisations) and 2 (deaths) we show the relationships between twenty-five baseline characteristics and the ALS outcomes. After adjustment for selected confounding factors, being older (hazard ratio per 10 year increase; 95\% confidence interval: $1.92 ; 1.58,2.33)$ and male $(1.37 ; 1.00$, 1.87) were associated with elevated rates of hospitalisation for ALS. Similar effects were apparent when death ascribed to the disorder was the outcome of interest. Of the remaining 23 potential risk indices, however, there was only a suggestion that taller people experienced an increased risk of hospitalisation (per SD increase: $1.31 ; 1.09,1.59$ ); weaker effects were apparent in the mortality analyses $(1.21 ; 0.98,1.48)$. While there was some indication of an elevated rate of hospitalisations in people who reported cigarette smoking, confidence intervals included unity and there was marked attenuation after adjustment for covariates which included socioeconomic status and health behaviours $(1.10 ; 0.73,1.66)$.

\section{Discussion}

The main finding of the present study was that there was no clear evidence of an association between ALS and the twenty-five risk indicators. The exceptions were the two most established risk factors - age and sex; that these associations were recapitulated here gives us some confidence in the generally negative results for the remainder of the baseline characteristics. These null findings are in keeping with observations from systematic reviews of risk factors for $\operatorname{ALS}^{17,20,41,42}$ and those for other neurodegenerative disorders such as dementia. ${ }^{43-45}$

The positive association between height and ALS also apparent for cancer $^{46,47}$ - potentially implicates early life exposures in the occurrence of this condition. The same has been speculated for dementia, ${ }^{48}$ although a reverse gradient to that seen herein has been reported. ${ }^{49}$ While under a degree of genetic control, relative to their shorter counterparts, taller people have, on average, been exposed to a more favourable pre-adult environment, which includes an optimal diet, fewer bouts of illness, 
Table I Hazard Ratios for the Association Between Baseline Characteristics and later Hospitalisation for Amyotrophic Lateral Sclerosis, UK Biobank 2006 to 2020

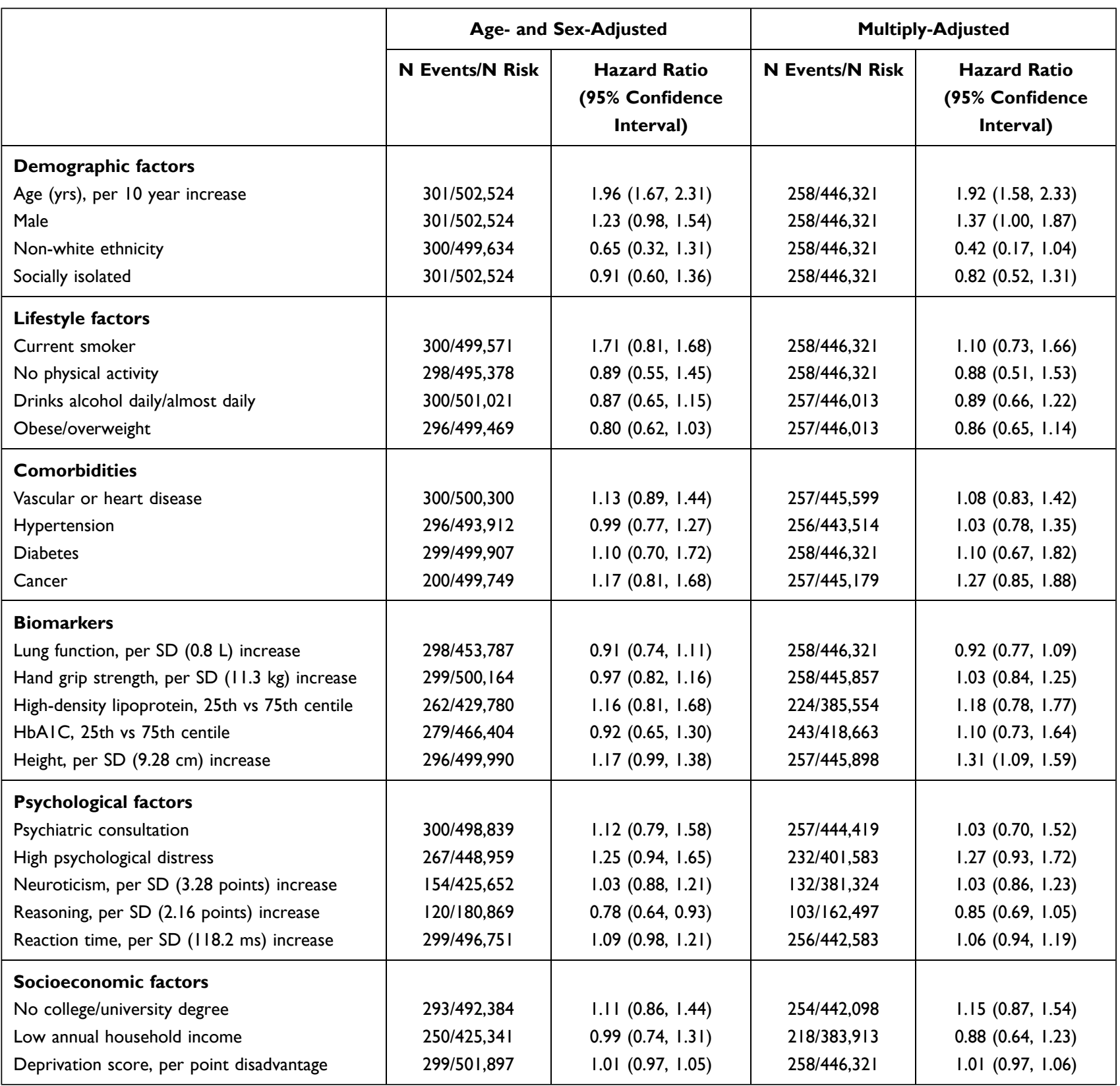

Notes: Multiple adjustment is adjustment for age, sex, ethnicity, smoking status, physical activity, diabetes, lung function, and deprivation score.

more affluent social circumstances, and a lower stress household, ${ }^{50}$ though we are unable to identify which, if any, of these correlates may be exerting an influence on ALS risk.

\section{Study Strengths and Weaknesses}

The present study has its strengths, including its size which facilitates the accumulation of a sufficiently high number of cases for analyses of a rare health outcome alongside left-censoring to take into account reverse causality, and the well characterised nature of the study participants. Further, in the aetiological literature, investigators often focus on the predictive value of a single characteristic rather than take a comprehensive approach, as we have, so facilitating cross-comparison. Inevitably, however, our work has several weaknesses. First, the present study sample comprises only the $5.5 \%$ of the target population who agreed to participate. ${ }^{51}$ As has been demonstrated, ${ }^{52,53}$ the data material is therefore inappropriate for estimation of risk factor or disease prevalence 
Table 2 Hazard Ratios for the Association Between Baseline Characteristics and Death from Amyotrophic Lateral Sclerosis, UK Biobank 2006 to 2020

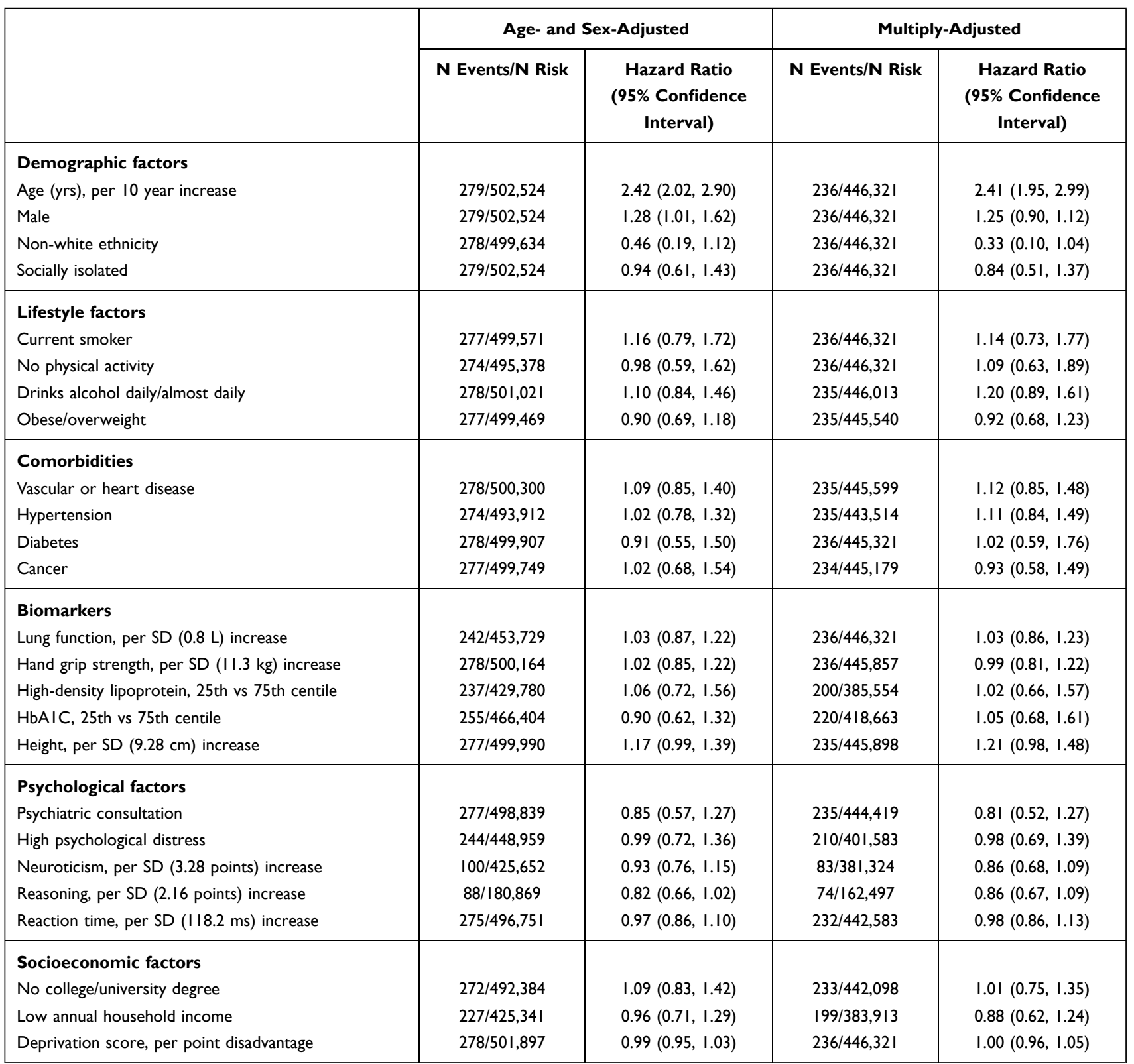

Notes: Multiple adjustment is adjustment for age, sex, ethnicity, smoking status, physical activity, diabetes, lung function, and deprivation score.

and for event incidence, including presumably ALS. These observations do not, however, seem to influence reproducibility of the association of established risk factors for non-communicable disease such as vascular disease and selected cancers, and other health endpoints such as suicide and selected cancers. ${ }^{53}$ We think the same reasoning can be applied to the present disease outcome.

Second, while we set out to further test the relation of selected risk factors (e.g., cigarette smoking, diabetes), and potentially identify novel ones (e.g., cognitive function, psychological distress), several other factors (e.g., alcohol intake, extant cancer, blood lipids) were not hypothesisdriven. Third, baseline data are certainly time-varying in the period between study induction and end of follow-up. This is a perennial issue in cohort studies and one we were able to investigate using data from a resurvey that took place around 8 years after baseline examination in a representative sub-sample of participants. ${ }^{37,54}$ Analyses revealed moderate to high stability for some covariates, including education $(\mathrm{r}=0.86, \mathrm{p}<0.001, \mathrm{~N}=30,350)$ and body mass index 
$(\mathrm{r}=0.90, \mathrm{p}<0.001, \mathrm{~N}=34,662)$, whereas the magnitude was somewhat lower for co-morbidities such as diabetes $(\mathrm{r}=0.63$, $\mathrm{P}<0.001, \mathrm{~N}=31,037)$ and serious mental illness $(\mathrm{r}=0.64, \mathrm{p}<$ $0.001, \mathrm{~N}=47,291)$, as it was for cigarette smoking ( $\mathrm{r}=0.60$, $\mathrm{p}<0.001, \mathrm{~N}=31,037)$. Lastly, we did not have data on event onset, only in-patient hospitalisation and death.

In conclusion, other than the known associations for age and sex, we did not find convincing evidence of links with ALS for other risk indices in the present analyses.

\section{Access to Data}

Data from UK Biobank (http://www.ukbiobank.ac.uk/) are available to bona fide researchers upon application. This research has been conducted under application 10279 .

\section{Acknowledgments}

We thank the participants in UK Biobank for their forbearance.

\section{Funding}

GDB is supported by the Medical Research Council (MR/ P023444/1) and the US National Institute on Aging (1R56AG052519-01; 1R01AG052519-01A1).

\section{Disclosure}

The authors report no conflicts of interest in this work.

\section{References}

1. Byrne S, Elamin M, Bede P, et al. Cognitive and clinical characteristics of patients with amyotrophic lateral sclerosis carrying a C9orf72 repeat expansion: a population-based cohort study. Lancet Neurol. 2012;11(3):232-240. doi:10.1016/S1474-4422(12)70014-5

2. Abhinav K, Stanton B, Johnston C, et al. Amyotrophic lateral sclerosis in South-East England: a population-based study. The South-East England register for amyotrophic lateral sclerosis (SEALS Registry). Neuroepidemiology. 2007;29(1-2):44-48. doi:10.1159/000108917

3. Marin B, Boumédiene F, Logroscino G, et al. Variation in worldwide incidence of amyotrophic lateral sclerosis: a meta-analysis. Int J Epidemiol. 2017;46(1):57-74.

4. Chiò A, Mora G, Moglia C, et al. Secular trends of amyotrophic lateral sclerosis: the Piemonte and valle d'aosta register. JAMA Neurol. 2017;74(9):1097-1104. doi:10.1001/jamaneurol.2017.1387

5. Armon C. An evidence-based medicine approach to the evaluation of the role of exogenous risk factors in sporadic amyotrophic lateral sclerosis. Neuroepidemiology. 2003;22(4):217-228. doi:10.1159/000070562

6. Zhang L, Tang L, Huang T, Fan D. Life course adiposity and amyotrophic lateral sclerosis: a Mendelian randomization study. Ann Neurol. 2020;87(3):434-441. doi:10.1002/ana.25671

7. Nakken O, Meyer HE, Stigum H, Holmøy T. High BMI is associated with low ALS risk: a population-based study. Neurology. 2019;93(5): e424-e32. doi:10.1212/WNL.0000000000007861

8. Alonso A, Logroscino G, Hernán MA. Smoking and the risk of amyotrophic lateral sclerosis: a systematic review and meta-analysis. J Neurol Neurosurg Psychiatry. 2010;81(11):1249-1252. doi:10.1136/ jnnp.2009.180232
9. Zhan Y, Fang F. Smoking and amyotrophic lateral sclerosis: a Mendelian randomization study. Ann Neurol. 2019;85(4):482-484. doi:10.1002/ana.25443

10. Wang MD, Little J, Gomes J, Cashman NR, Krewski D. Identification of risk factors associated with onset and progression of amyotrophic lateral sclerosis using systematic review and meta-analysis. Neurotoxicology. 2017;61:101-130.

11. D’Ovidio F, Rooney JPK, Visser AE, et al. Association between alcohol exposure and the risk of amyotrophic lateral sclerosis in the Euro-MOTOR study. J Neurol Neurosurg Psychiatry. 2019;90(1):11-19.

12. Meng E, Yu S, Dou J, et al. Association between alcohol consumption and amyotrophic lateral sclerosis: a meta-analysis of five observational studies. Neurol Sci. 2016;37(8):1203-1208. doi:10.1007/ s10072-016-2575-0

13. Roberts AL, Johnson NJ, Chen JT, Cudkowicz ME, Weisskopf MG. Race/ethnicity, socioeconomic status, and ALS mortality in the United States. Neurology. 2016;87(22):2300-2308. doi:10.1212/ WNL.0000000000003298

14. Åberg M, Nyberg J, Robertson J, et al. Risk factors in Swedish young men for amyotrophic lateral sclerosis in adulthood. $J$ Neurol. 2018;265(3):460-470. doi:10.1007/s00415-017-8719-1

15. Tsai CP, Lee JK, Lee CT. Type II diabetes mellitus and the incidence of amyotrophic lateral sclerosis. J Neurol. 2019;266(9):2233-2243. doi:10.1007/s00415-019-09405-x

16. D'Ovidio F, d'Errico A, Carnà $P$, Calvo $A$, Costa $G$, Chiò $A$. The role of pre-morbid diabetes on developing amyotrophic lateral sclerosis. Eur J Neurol. 2018;25(1):164-170. doi:10.1111/ene.13465

17. Al-Chalabi A, Hardiman O. The epidemiology of ALS: a conspiracy of genes, environment and time. Nat Rev Neurol. 2013;9 (11):617-628. doi:10.1038/nrneurol.2013.203

18. Rusina R, Vandenberghe R, Bruffaerts R. Cognitive and behavioral Manifestations in ALS: beyond Motor System Involvement. Diagnostics. 2021;11(4):548.

19. Chiò A, Logroscino G, Hardiman O, et al. Prognostic factors in ALS: a critical review. Amyotroph Lateral Scler. 2009;10(5-6):310-323. doi:10.3109/17482960802566824

20. Couratier P, Corcia P, Lautrette G, Nicol M, Preux PM, Marin B. Epidemiology of amyotrophic lateral sclerosis: a review of literature. Rev Neurol (Paris). 2016;172(1):37-45. doi:10.1016/j. neurol.2015.11.002

21. von Elm E, Altman DG, Egger M, et al. The strengthening the reporting of observational studies in epidemiology (STROBE) statement: guidelines for reporting observational studies. Lancet. 2007;370(9596):1453-1457. doi:10.1016/S0140-6736(07)61602-X

22. Lassale C, Gaye B, Hamer M, Gale CR, Batty GD. Ethnic disparities in hospitalisation for COVID-19 in England: the role of socioeconomic factors, mental health, and inflammatory and pro-inflammatory factors in a community-based cohort study. Brain Behav Immun. 2020;88:44-49. doi:10.1016/j.bbi.2020.05.074

23. Elovainio M, Hakulinen C, Pulkki-Raback L, et al. Contribution of risk factors to excess mortality in isolated and lonely individuals: an analysis of data from the UK Biobank cohort study. Lancet Public Health. 2017;2(6):e260-e6. doi:10.1016/S2468-2667(17)30075-0

24. Batty GD, Deary IJ, Luciano M, Altschul DM, Kivimaki M, Gale CR. Psychosocial factors and hospitalisations for COVID-19: prospective cohort study based on a community sample. Brain Behav Immun. 2020;89:569-578. doi:10.1016/j.bbi.2020.06.021

25. Hamer M, Kivimaki M, Gale CR, Batty GD. Lifestyle risk factors, inflammatory mechanisms, and COVID-19 hospitalization: a community-based cohort study of 387,109 adults in UK. Brain Behav Immun. 2020;87:184-187. doi:10.1016/j.bbi.2020.05.059

26. Celis-Morales CA, Lyall DM, Steell L, et al. Associations of discretionary screen time with mortality, cardiovascular disease and cancer are attenuated by strength, fitness and physical activity: findings from the UK Biobank study. BMC Med. 2018;16(1):77. doi:10.1186/ s12916-018-1063-1 
27. Hamer M, Gale CR, Kivimaki M, Batty GD. Overweight, obesity, and risk of hospitalization for COVID-19: a community-based cohort study of adults in the United Kingdom. Proc Natl Acad Sci U S A. 2020;117(35):21011-21013. doi:10.1073/pnas.2011086117

28. Chobanian AV, Bakris GL, Black HR, et al. The seventh report of the joint national committee on prevention, detection, evaluation, and treatment of high blood pressure: the JNC 7 report. JAMA. 2003;289(19):2560-2572. doi:10.1001/jama.289.19.2560

29. Batty GD, Hamer M. Vascular risk factors, Framingham risk score, and COVID-19: community-based cohort study. Cardiovasc Res. 2020;116(10):1664-1665. doi:10.1093/cvr/cvaa178

30. Hamer M, Gale CR, Batty GD. Diabetes, glycaemic control, and risk of COVID-19 hospitalisation: population-based, prospective cohort study. Metabolism. 2020;112:154344. doi:10.1016/j.metabol.20 20.154344

31. Batty GD, McIntosh AM, Russ TC, Deary IJ, Gale CR. Psychological distress, neuroticism, and cause-specific mortality: early prospective evidence from UK Biobank. $J$ Epidemiol Community Health. 2016;70(11):1136-1139. doi:10.1136/jech-2016207267

32. Kroenke K, Spitzer RL, Williams JB, Lowe B. An ultra-brief screening scale for anxiety and depression: the PHQ-4. Psychosomatics. 2009;50(6):613-621.

33. Lowe B, Wahl I, Rose M, et al. A 4-item measure of depression and anxiety: validation and standardization of the Patient Health Questionnaire-4 (PHQ-4) in the general population. J Affect Disord. 2010;122(1-2):86-95. doi:10.1016/j.jad.2009.06.019

34. Deary IJ, Bedford A. Some origins and evolution of the EPQ-R (short form) Neuroticism and Extraversion items. Pers Individ Dif. 2011;50 (8):1213-1217. doi:10.1016/j.paid.2011.02.011

35. Gale CR, Čukić I, Batty GD, McIntosh AM, Weiss A, Deary IJ. When is higher neuroticism protective against death? Findings from UK Biobank. Psychol Sci. 2017;28(9):1345-1357. doi:10.1177/ 0956797617709813

36. Davies G, Marioni RE, Liewald DC, et al. Genome-wide association study of cognitive functions and educational attainment in UK Biobank (N=112 151). Mol Psychiatry. 2016;21(6):758-767. doi:10.1038/mp.2016.45

37. Batty GD, Deary IJ, Gale CR. Pre-pandemic cognitive function and COVID-19 mortality: prospective cohort study. Eur J Epidemiol. 2021;36(5):559-564. doi:10.1007/s10654-021-00743-7

38. Deary IJ, Der G, Ford G. Reaction times and intelligence differences: a population-based cohort study. Intelligence. 2001;29:389-399. doi:10.1016/S0160-2896(01)00062-9

39. Abell JG, Lassale C, Batty GD, Zaninotto P. Risk factors for hospital admission after a fall: a prospective cohort study of community-dwelling older people. J Gerontol a Biol Sci Med Sci. 2021;76(4):666-674. doi:10.1093/gerona/glaa255

40. Cox DR. Regression models and life-tables. $J R$ Stat Soc. 1972;34:187-220.

41. Ingre C, Roos PM, Piehl F, Kamel F, Fang F. Risk factors for amyotrophic lateral sclerosis. Clin Epidemiol. 2015;7:181-193.
42. Oskarsson B, Gendron TF, Staff NP. Amyotrophic lateral sclerosis: an update for 2018. Mayo Clin Proc. 2018;93(11):1617-1628. doi:10.1016/j.mayocp.2018.04.007

43. Batty GD, Russ TC, Starr JM, Stamatakis E, Kivimaki M. Modifiable cardiovascular disease risk factors as predictors of dementia death: pooling of ten general population-based cohort studies. $J$ Negat Results Biomed. 2014;13:8. doi:10.1186/1477-5751-13-8

44. Killin LO, Starr JM, Shiue IJ, Russ TC. Environmental risk factors for dementia: a systematic review. BMC Geriatr. 2016;16(1):175. doi:10.1186/s12877-016-0342-y

45. Bellou V, Belbasis L, Tzoulaki I, Middleton LT, Ioannidis JPA, Evangelou E. Systematic evaluation of the associations between environmental risk factors and dementia: an umbrella review of systematic reviews and meta-analyses. Alzheimers Dement. 2017;13 (4):406-418. doi:10.1016/j.jalz.2016.07.152

46. Batty GD, Barzi F, Woodward M, et al. Adult height and cancer mortality in Asia: the Asia Pacific Cohort Studies Collaboration. Ann Oncol. 2010;21(3):646-654. doi:10.1093/annonc/mdp363

47. Lee CM, Barzi F, Woodward M, et al. Adult height and the risks of cardiovascular disease and major causes of death in the Asia-Pacific region: 21,000 deaths in 510,000 men and women. Int J Epidemiol. 2009;38(4):1060-1071. doi:10.1093/ije/dyp150

48. Whalley LJ, Dick FD, McNeill G. A life-course approach to the aetiology of late-onset dementias. Lancet Neurol. 2006;5(1):87-96. doi:10.1016/S1474-4422(05)70286-6

49. Russ TC, Kivimaki M, Starr JM, Stamatakis E, Batty GD. Height in relation to dementia death: individual participant meta-analysis of 18 UK prospective cohort studies. $B r \quad J$ Psychiatry. 2014;205 (5):348-354. doi:10.1192/bjp.bp.113.142984

50. Batty GD, Shipley MJ, Gunnell D, et al. Height, wealth, and health: an overview with new data from three longitudinal studies. Econ Hum Biol. 2009;7(2):137-152. doi:10.1016/j.ehb.2009.06.004

51. Sudlow C, Gallacher J, Allen N, et al. UK biobank: an open access resource for identifying the causes of a wide range of complex diseases of middle and old age. PLoS Med. 2015;12(3):e1001779. doi:10.1371/journal.pmed.1001779

52. Fry A, Littlejohns TJ, Sudlow C, et al. Comparison of Sociodemographic and Health-Related Characteristics of UK Biobank Participants With Those of the General Population. Am J Epidemiol. 2017;186(9):1026-1034. doi:10.1093/aje/kwx246

53. Batty GD, Gale CR, Kivimäki M, Deary IJ, Bell S. Comparison of risk factor associations in UK Biobank against representative, general population based studies with conventional response rates: prospective cohort study and individual participant meta-analysis. $B M J$. 2020;1:368.

54. Lassale C, Hamer M, Hernáez Á, Gale CR, Batty GD. Association of pre-pandemic high-density lipoprotein cholesterol with risk of COVID-19 hospitalisation and death: the UK Biobank cohort study. Prev Med Rep. 2021;23:101461. doi:10.1016/j.pmedr.2021.101461
Clinical Epidemiology

\section{Publish your work in this journal}

Clinical Epidemiology is an international, peer-reviewed, open access, online journal focusing on disease and drug epidemiology, identification of risk factors and screening procedures to develop optimal preventative initiatives and programs. Specific topics include: diagnosis, prognosis, treatment, screening, prevention, risk factor modification,

Submit your manuscript here: https://www.dovepress.com/clinical-epidemiology-journal systematic reviews, risk \& safety of medical interventions, epidemiology \& biostatistical methods, and evaluation of guidelines, translational medicine, health policies \& economic evaluations. The manuscript management system is completely online and includes a very quick and fair peer-review system, which is all easy to use. 